\title{
Systematic literature review on search based software testing
}

\begin{abstract}
The use of random search is very poor at finding solutions when those solutions occupy a very small part of the overall search space. Test data may be found faster and more reliably if the search is given some guidance. This work is a paper that explains the application of metaheuristic techniques in search-based software testing. The paper systematically review 47 papers selected randomly from online databases and conference proceeding based on the metaheuristic search techniques that have been most widely applied to problem solving, the different fitness function used for test data selection in each of the metaheuristic technique, and the limitation in the use of each search-based technique for software testing. It was found that GA outperformed its counterparts SA, HC, GP and random search approaches in generating test data automatically, different approaches were used to make sure that test data are selected within shorter period of time and also with wider coverage of the paths based on the fitness function, and most of the limitations of the articles are the handling of complex data types, like array, object types, and branch coverage. The paper also provides areas of possible future work on the use of metaheuristic techniques in search-based software testing.
\end{abstract}

Keyword: Metaheuristic techniques; Search-based software testing; Software testing; Systematic literature review 